Check for updates

Cite this: RSC Adv., 2019, 9, 29331

\title{
The directions of an external electric field control the catalysis of the hydroboration of $\mathrm{C}-\mathrm{O}$ unsaturated compounds $\uparrow$
}

\begin{abstract}
Ming-Xia Zhang, ${ }^{a}$ Hong-Liang Xu (D) *a and Zhong-Min Su (D) ab
The orientation directions of an external electric field (EEF) in catalyzing chemical reactions are an important factor because they can significantly accelerate reaction activity. In this study, we explored a new antiMarkovnikov hydroboration reaction of $\mathrm{C}-\mathrm{O}$ unsaturated compounds (e.g., benzaldehyde and benzophenone) with the aim of revealing the dominant direction of EEF in accelerating the reactions, and pinacolborane (HBpin) was selected as an efficient reductant. The calculation results showed that the EEF oriented along the direction of electron pair transform rather than that of the molecular dipole moment could reduce the barrier of the hydroboration of benzaldehyde by $20 \mathrm{kcal} \mathrm{mol}^{-1}$ when the EEF was up to $150 \times 10^{-4}$ au. Moreover, the Markovnikov hydroboration of aldehyde and ketone was investigated for obtaining the mechanistic-switchover point. Unsatisfactorily, the EEF could just influence the respective barriers without a promising competition with the anti-Markovnikov hydroboration reactions.
\end{abstract}

Received 23rd May 2019

Accepted 29th August 2019

DOI: $10.1039 / \mathrm{c} 9 \mathrm{ra} 03895 \mathrm{~g}$

rsc.li/rsc-advances

concise, efficient, and environmentally friendly methods for hydroboration; for example, a catalyst-free and solvent-free hydroboration reaction of aldehydes has been reported recently. ${ }^{11}$ Herein, we focused our attention on the development of a greener catalyst for the catalytic hydroboration of aldehydes and ketones.

The external electric field (EEF) is known as a smart reagent in chemistry, which can act on atoms, molecules and complex matter with a range of phenomena. ${ }^{12}$ For example, by orienting the field along the "reaction axis", the direction of the "electron pair transform" will catalyse the reaction. Moreover, flipping the field's orientation along the reaction-axis will cause inhibition of the reaction. The harnessing of EEF for controlling the reactivity and selectivity of chemical reactions has gained significant attention ${ }^{13}$ since the electrostatic catalysis of bond cleavage has been reported to occur in highly concentrated $\mathrm{LiClO}_{4}$ solutions. ${ }^{14}$ Especially, one of the theoretical study on the Diels-Alder reaction ${ }^{15}$ catalyzed by the EEF has been successfully verified by experiment ${ }^{16}$ using a surface model system coupled with a scanning tunnelling microscopy breakjunction approach ${ }^{17}$ in 2016; this has immensely enhanced the possibility and reliability of the EEF as a catalyst. The inspection of these abovementioned studies has revealed that the orientation direction of the EEF is an important influencing factor because it may control the reaction reactivity or selectivity by acting on the direction of electron pair transform or molecular dipole moment; although one study had been reported on the importance of the molecular dipole moment induced by oriented-EEFs in the Diels-Alder reactions, ${ }^{13 e}$ we herein explored the role of the EEF in catalyzing a new chemical
${ }^{a}$ Institute of Functional Material Chemistry, Department of Chemistry, National \& Local United Engineering Lab for Power Battery, Northeast Normal University, Changchun 130024, Jilin, People's Republic of China. E-mail: hlxu@nenu.edu.cn ${ }^{b}$ School of Chemistry and Environmental Engineering, Changchun University of Science and Technology, Changchun, Jilin 130012, P. R. China

$\dagger$ Electronic supplementary information (ESI) available. See DOI: 10.1039/c9ra03895g 
reaction with the aim of revealing the dominant direction in accelerating the reaction activity. Pioneered by the studies on the hydroboration of compounds containing $\mathrm{C}-\mathrm{C}$ and $\mathrm{C}-\mathrm{N}$ multiple-bonds in the presence of EEF, ${ }^{18}$ the original hydroboration of $\mathrm{C}-\mathrm{O}$ unsaturated compounds (Scheme 1) was studied first, in which pinacolborane (HBpin) was selected as an efficient reductant with EEF as the catalyst.

As is well-known, in the anti-Markovnikov hydroboration reaction, a B-containing group is added to the terminal atom of the unsaturated bond, which generates a linear structure. On the other hand, the $\mathrm{B}$ atom is added to the corresponding internal atom, and it provides a branched Markovnikov organoborane. Recently, the Markovnikov hydroboration of alkenes has gained significant attention. ${ }^{19}$ Moreover, a proper EEF may make the Markovnikov hydroboration reaction easier, and even it becomes the primary addition reaction. ${ }^{18}$ As a result, we first studied the Markovnikov hydroboration reaction of an aldehyde and a ketone to verify this protocol, in which the experimental molecules benzaldehyde and benzophenone (Scheme 1) were selected. According to the results, the EEF could influence the Markovnikov hydroboration of the aldehyde and ketone, but only a small scale of decrease was induced as far as the barrier heights of these reactions were concerned. Specifically, the Markovnikov hydroboration reaction could still not compete with the anti-Markovnikov hydroboration reaction. Therefore, the detailed computation results are shown in the ESI (Fig. S1 and S2; Tables S1 and S2†).

Hereinafter, we have comprehensively discussed the effects of the EEF on the anti-Markovnikov hydroboration reaction of $\mathrm{C}-\mathrm{O}$ unsaturated compounds. At first, all the stationary points along the potential energy surface were optimized under the field-free conditions using the B3LYP $\operatorname{method}^{20}$ with the basis set of $6-31 G^{*}$, and then, a larger level of $6-311++\mathrm{G}^{* *}$ was used for the calculations of single-point energies. After this, we focused on the effects of the EEF on these reactions. The directions of the coordinate axes and the positive $Z$-direction $\left(F_{Z}\right)$ of the EEF are shown in Scheme 1. The structures (Fig. S3 and S4 $\dagger$ ) of these stationary points at various field strengths $\left( \pm 25, \pm 50\right.$ and $\pm 75 \times 10^{-4}$ au) from three directions $\left(F_{X}, F_{Y}\right.$, and $\left.F_{Z}\right)$ were re-optimized, and their single-point energies (Tables $\mathrm{S} 3$ and $\mathrm{S} 4 \dagger$ ) were also recalculated. To check that the trends are not an artifact of the methodology, several functionals (such as PBE0, M06, and BP86) combined with a double or a triple basis set were

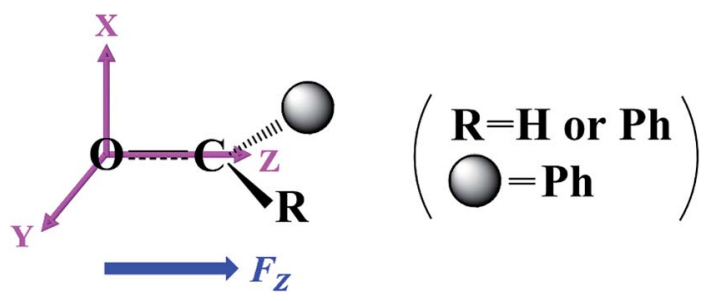

Scheme 1 Selected model molecules of benzaldehyde and benzophenone and the definitions of the directions of the coordinate axes and positive $Z$-direction $\left(F_{Z}\right)$ of the EEF. used for checking the calculations. The corresponding results are shown in the ESI (Tables S5-S16 and Fig. S5-S7†). In addition, the THF solvent with the $\mathrm{SMD}^{21}$ model was introduced to further examine the effect of solvents on the hydroboration reactions (Tables S17, S18 and Fig. S8†). The Gaussian 09 program ${ }^{22}$ was employed for all the calculations in the present study.

From Fig. 1a, we can deduce that the hydroboration reaction of benzaldehyde with HBpin needs to overcome a barrier of $34.6 \mathrm{kcal} \mathrm{mol}^{-1}$ to produce a linear organoborane under the fieldfree conditions. Fig. 1b exhibits the barriers of this antiMarkovnikov hydroboration reaction at different field strengths from different directions, in which the red, green and black lines correspond to the barriers when the EEF is oriented along the $F_{X} ; F_{Y}$; and $F_{Z}$ axes. Obviously, (i) the green line $\left(F_{Y}\right)$ has a small variation whether the orientation is along the positive or negative $Y$-axis direction because this direction is oriented perpendicular to the paper plane, which is neither the direction of the electron pair transform nor the direction of the molecular dipole moment. For example, upon analyzing its dipole moment (Fig. 2a), we could clearly know that only a minor component was located in the $Y$-axis. This is also the reason that the variations in the dipole moments (Table 1) of $F_{Y}(Z)$ slightly changes (the biggest change is only $0.26 \mathrm{D}$ ) with a change in the EEF. Thus, the barriers of the anti-Markovnikov hydroboration of benzaldehyde had a small variation as the EEF was oriented along $\pm F_{Y}$ (ii) When the EEF was increased along $-F_{X}$, the barriers (red line) also increased gradually. On the other hand, when the EEF was increased along $F_{X}$, the barriers decreased. In line with the rules, ${ }^{12} F_{X}$ is the direction of electron pair transform. Fig. 3 shows the electron-density difference (EDD) maps ${ }^{23}$ of the transition state (TS1) at various EEFs. We can conclude that the electron densities of the $\mathrm{H}$ atom deplete (the blue dash line) as the EEF is varied from -25 to $-75 \times 10^{-4}$ au, which inhibits the addition of benzaldehyde with HBpin. Even the charges of the $\mathrm{H}$ atom flipped to a positive value $(0.022)$ when $F_{X}=-75 \times 10^{-4}$ au. On the contrary, the electron densities of the $\mathrm{H}$ atom increased (the solid red line) as the EEF varied from 25 to $75 \times 10^{-4}$ au. Therefore, the barriers of this hydroboration along $F_{X}$ decreased. Thus, although the electron densities of the $\mathrm{O}$ atom underwent a big change, the $\mathrm{H}$ atom acted as a dominant factor. Comparatively, the dipole moments (Table 1) of $F_{X}(Z)$ changed slightly in the entire field region. (iii) Furthermore, the inspection of the black line $\left(F_{Z}\right)$ in Fig. $1 \mathrm{~b}$ revealed that the variation tendency of the barriers was contrary to that in the case of the red line $\left(F_{X}\right)$. We attributed this to the changes in dipole moments with the EEF because the dipole moment interactions were quite important in the reactions involving borane or its derivatives. ${ }^{24}$ From Fig. 2a, we can see that the major component of the dipole moment is located in the $Z$-axis, that is, the direction of $F_{Z}$. The dipole moments (Table 1) of $F_{Z}(Z)$ were found to gradually increase from 2.98 (0) to $4.81 \mathrm{D}(-75 \times$ $10^{-4} \mathrm{au}$ ), which indicated that the charges transferred to the $\mathrm{O}$ atom from the $\mathrm{C}$ atom. Consequently, the barriers of this hydroboration reaction along $-F_{Z}$ decreased and vice versa.

To our surprise and interest, when the field strengths were small, the variation extents of the barriers induced by EEF from $F_{X}$ and $F_{Z}$ were almost equal (Fig. 1b). However, the red line $\left(F_{X}\right)$ had a large decrease when EEF $>|75| \times 10^{-4}$ au when compared with the black 
a)

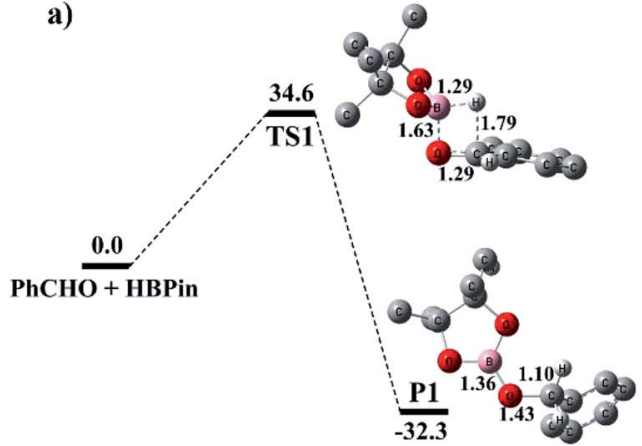

b)

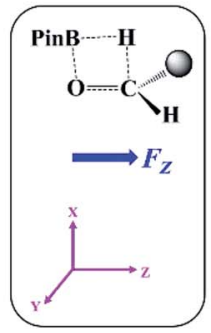

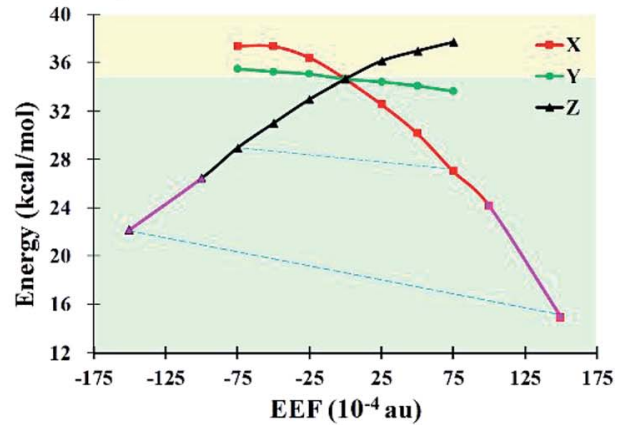

Fig. 1 (a) Relative energies ( $\mathrm{kcal} \mathrm{mol}^{-1}$ ) of the stationary points in the hydroboration of benzaldehyde with HBpin in the absence of EEF. (b) The barriers of these hydroboration reactions when the EEFs were $\pm 25, \pm 50$ and $\pm 75 \times 10^{-4}$ au in three different directions i.e., $F_{X}, F_{Y}$, and $F_{Z}$, respectively.

line $\left(F_{Z}\right)$. To further examine this conclusion, we added two points $\left(100\right.$ and $\left.150 \times 10^{-4} \mathrm{au}\right)$ for the anti-Markovnikov hydroboration of benzaldehyde. The results are also shown in Fig. 1b in the form of the added pink lines. Noticeably, not only the reduced tendency was much obvious, but also the barrier height was found to be reduced to only $14.9 \mathrm{kcal} \mathrm{mol}^{-1}$ in the hydroboration of benzaldehyde as $F_{X}$ $=150 \times 10^{-4}$ au. Based on our previous discussion, $F_{X}$ is the direction of electron pair transform, and $F_{Z}$ is almost the direction of molecular dipole moment. Thus, from this study, we could conclude that when the EEF strength was small, the effects of the EEF on this hydroboration of benzaldehyde were almost equal regardless of the direction of the electron pair transform or molecular dipole moment. On the other hand, when the EEF was large, the direction of the electron pair transform might be the dominant influencing factor. However, this significant reduction demonstrates that the EEF is a good catalyst for the hydroboration of benzaldehyde with HBpin.

To further explore the effects of the EEF on the hydroboration of a ketone, we investigated the hydroboration of benzophenone with HBpin at the same level as that of benzaldehyde. In Fig. 4a, it can be observed that the barrier of this reaction is $35.2 \mathrm{kcal} \mathrm{mol}^{-1}$ under the field-free conditions. When different EEFs from different directions were imposed on this hydroboration, as shown in Fig. 4b, an interesting phenomenon occurred. Except for the obvious effects of $F_{X}$ and $F_{Z}$ on these barriers, the variation tendencies were found to be similar to that of benzaldehyde (Fig. 1b) because benzophenone could be regarded as one of the derivatives of benzaldehyde, that is, the $\mathrm{H}$ atom in
Table 1 Dipole moment (D) of benzaldehyde when EEF $\left(10^{-4} \mathrm{au}\right)$ is oriented along $F_{Y}$ and $F_{Z}$

\begin{tabular}{llllllll}
\hline$\mu$ & -75 & -50 & -25 & 0 & 25 & 50 & 75 \\
$F_{X}(Z)$ & 2.75 & 2.89 & 2.95 & 2.98 & 3.02 & 2.98 & 2.99 \\
$F_{Y}(Z)$ & 2.74 & 2.82 & 2.9 & 2.98 & 3.07 & 3.15 & 3.24 \\
$F_{Z}(Z)$ & 4.81 & 4.2 & 3.59 & 2.98 & 2.38 & 1.78 & 1.18
\end{tabular}

benzaldehyde was substituted by phenyl to form benzophenone. This is the reason that the influences of $F_{X}$ and $F_{Z}$ on the hydroboration of benzophenone are similar to those on the hydroboration of benzaldehyde. Moreover, a barrier higher in energy $\left(0.6 \mathrm{kcal} \mathrm{mol}^{-1}\right)$ than that in the case of benzaldehyde was significantly due to steric hindrance from phenyl. Note that the effect of $F_{Y}$ was different from that in the case of benzaldehyde, in which the blue curve was almost symmetric and the variation tendency was very small (Fig. 4b). We attribute this to the fact that benzophenone is a symmetric molecule (Fig. 2b), and the direction of the dipole moment is completely located at the axis of symmetry. Fig. 4c also shows the values of dipole moments with the changes in the EEF. It is observed that the variations are very small, and regardless of the positive or negative $Y$-direction, the dipole moments are slightly increased.

In summary, in the present study, at first, the effects of the external electric field (EEF) on the anti-Markovnikov hydroboration of benzaldehyde and benzophenone with HBpin were discussed, and further, the dominant direction of the EEF in catalyzing these reactions was explored. When the EEFs were a)

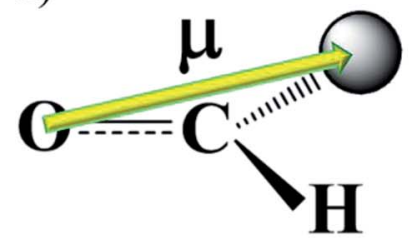

b)

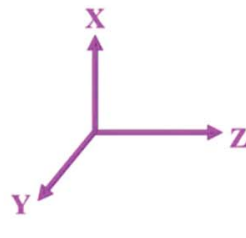

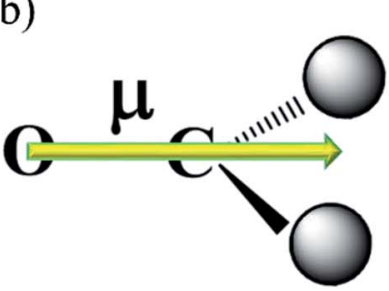

Fig. 2 (a) Direction of the dipole moment of benzaldehyde. (b) Direction of the dipole moment of benzophenone. 

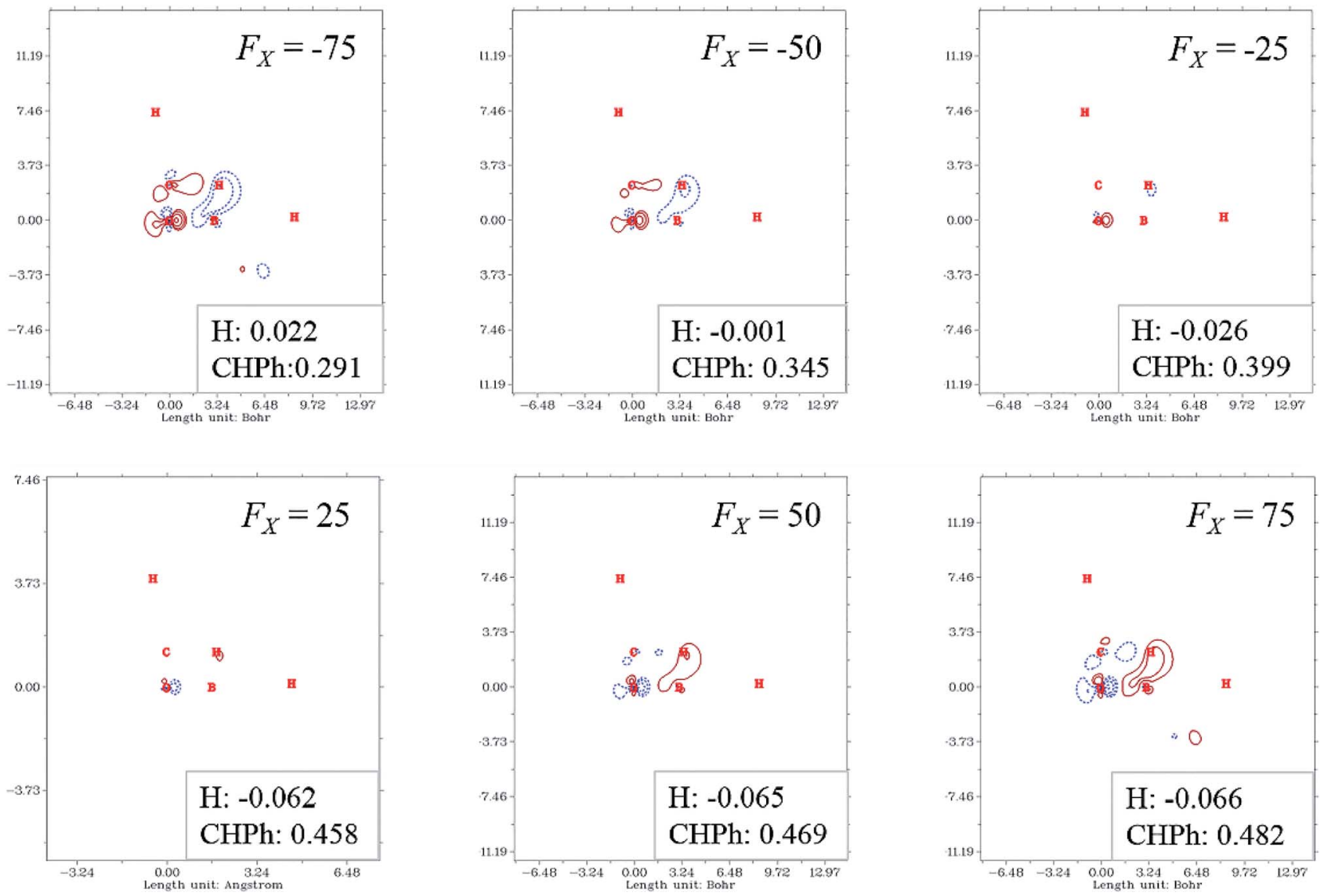

Fig. 3 Electron-density difference (EDD) maps of the transition state (TS1) at various EEFs $\left(\times 10^{-4} \mathrm{au}\right)$ with the charges of the $\mathrm{H}$ atom and $\mathrm{CHPh}$ group presented in the bottom right corner (the blue dash line represents the decreasing electron density, whereas the red solid line represents the increasing electron density; when $\mathrm{EEF}=0$, the charge density of the $\mathrm{H}$ atom is -0.050 and that of the $\mathrm{CHPh}$ group is 0.441 ).

oriented along $F_{X}$ or $-F_{Z}$, these reactions were catalyzed; otherwise, they were inhibited. Moreover, the small EEF induced equal effects on the reactions, whereas for the large $\mathrm{EEF}$, the effects from the direction of electron pair transform were more obvious than those from the molecular dipole moment. In addition, benzophenone could be considered as the analogue of substituted benzaldehyde. Thus, the EEF effects on the hydroboration of benzophenone were found to be similar to those on the hydroboration of benzaldehyde, except for the case of $F_{Y}$. The possible reason is that benzophenone is almost a symmetric molecule. Furthermore, the Markovnikov hydroboration of an aldehyde and a ketone was investigated. Unsatisfactorily, the EEF could just influence their barriers, but did not cause a mechanistic-switchover.
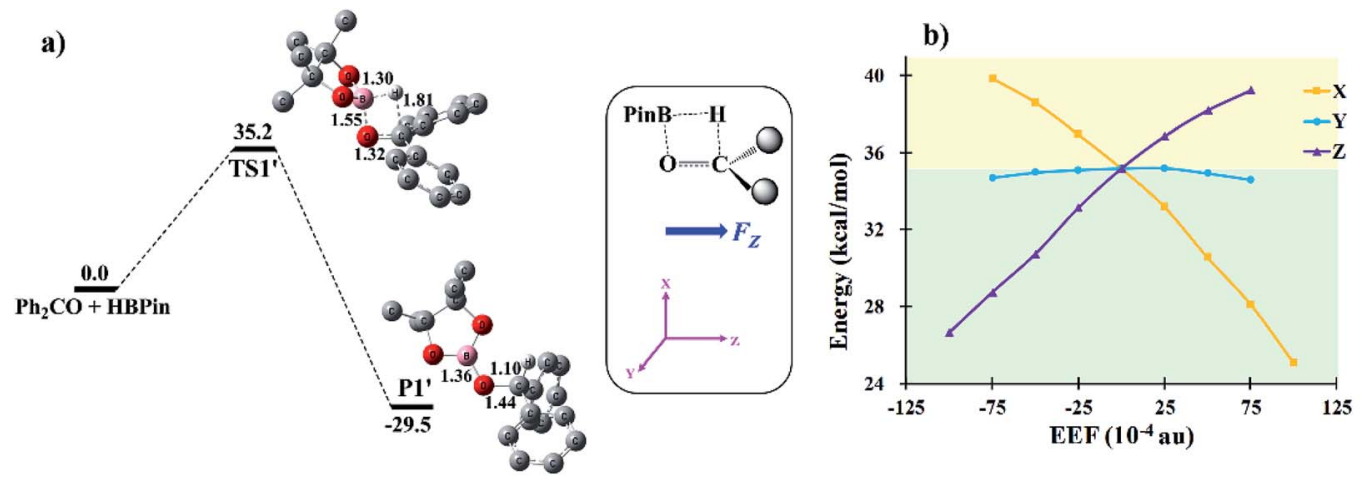

c)

\begin{tabular}{cc}
\hline$\mu$ & $F_{Y}(\mathrm{Z})$ \\
\hline-75 & 3.02 \\
-50 & 2.99 \\
-25 & 2.98 \\
0 & 2.98 \\
25 & 2.97 \\
50 & 3.01 \\
75 & 3.04 \\
\hline
\end{tabular}

Fig. 4 (a) Relative energies ( $\mathrm{kcal} \mathrm{mol}^{-1}$ ) of the stationary points in the hydroboration of benzophenone with HBpin in the absence of EEF; (b) the barriers of these hydroboration when the EEFs were $\pm 25, \pm 50$, and $\pm 75 \times 10^{-4}$ au from three directions i.e., $F_{X}, F_{Y}$, and $F_{Z}$, respectively; (c) dipole moments (D) of benzophenone when EEF was oriented along the $\pm Y$ direction. 


\section{Conflicts of interest}

There are no conflicts to declare.

\section{Acknowledgements}

The authors gratefully acknowledge the financial support received from the National Science Foundation of China (NSFC 21473026, 21503040) and the Science and Technology Development Planning of Jilin Province (20140101046JC), and H.-L. $\mathrm{X}$. acknowledges the support received from the project funded by the China Postdoctoral Science Foundation (2014M560227) and the Fundamental Research Funds for the Central Universities 2412018ZD008.

\section{References}

1 H. C. Brown and B. C. S. Rao, J. Am. Chem. Soc., 1956, 78, 5694-5695.

2 (a) S. J. Geier, C. M. Vogels and S. A. Westcott, in Boron Reagents in Synthesis, ed. A. Coca, American Chemical Society, New Haven, 2016, pp. 209-225; (b) J. Royes, A. B. Cuenca and E. Fernández, Eur. J. Org. Chem., 2018, 22, 2728-2739.

3 H. C. Brown, S. Krishnamurthy and N. M. Yoon, J. Org. Chem., 1976, 41, 1778-1791.

4 For selected reduction of carbonyl compounds to alcohols, see: (a) V. L. Weidner, C. J. Barger, M. Delferro, T. L. Lohr and T. J. Marks, ACS Catal., 2017, 7, 1244-1247; (b) Y. X. Liao and Q. S. Hu, J. Org. Chem., 2011, 76, 7602-7607; (c) K. Revunova and G. I. Nikonov, Dalton Trans., 2015, 44, 840-866; (d) C. C. Chong and R. Kinjo, ACS Catal., 2015, 5, 3238-3259; (e) B. T. Cho, Chem. Soc. Rev., 2009, 38, 443452; (f) J. Magano and J. R. Dunetz, Org. Process Res. Dev., 2012, 16, 1156-1184; (g) J.-i. Ito and H. Nishiyama, Tetrahedron Lett., 2014, 55, 3133-3146.

5 (a) S. R. Tamang and M. Findlater, J. Org. Chem., 2017, 82, 12857-12862; (b) S. R. Tamang, D. Bedi, S. ShafieiHaghighi, C. R. Smith, C. Crawford and M. Findlater, Org. Lett., 2018, 20, 6695-6700; (c) M. L. Shegavi, A. Baishya, K. Geetharani and S. K. Bose, Org. Chem. Front., 2018, 5, 3520-3525.

6 (a) R. Arévalo, C. M. Vogels, G. A. MacNeil, L. Riera, J. Pérez and S. A. Westcott, Dalton Trans., 2017, 46, 7750-7757; (b) R. J. Newland, J. M. Lynam and S. M. Mansell, Chem. Commun., 2018, 54, 5482-5485.

7 D. Yan, P. Dai, S. Chen, M. Xue, Y. Yao, Q. Shen and X. Bao, Org. Biomol. Chem., 2018, 16, 2787-2791.

8 T. Ghatak, K. Makarov, N. Fridman and M. S. Eisen, Chem. Commun., 2018, 54, 11001-11004.

9 (a) H. Osseili, D. Mukherjee, T. P. Spaniol and J. Okuda, Chem.-Eur. J., 2017, 23, 14292-14298; (b) Y. Wu, C. Shan, J. Ying, J. Su, J. Zhu, L. L. Liu and Y. Zhao, Green Chem., 2017, 19, 4169-4175.

10 (a) J. Schneider, C. P. Sindlinger, S. M. Freitag, H. Schubert and L. Wesemann, Angew. Chem., Int. Ed., 2017, 56, 333337; (b) V. A. Pollard, S. A. Orr, R. McLellan, A. R. Kennedy,
E. Hevia and R. E. Mulvey, Chem. Commun., 2018, 54, 1233-1236.

11 H. Stachowiak, J. Kaźmierczak, K. Kuciński and G. Hreczycho, Green Chem., 2018, 20, 1738-1742.

12 For selected special phenomena induced by external electric field, see: (a) S. Shaik, D. Mandal and R. Ramanan, Nat. Chem., 2016, 8, 1091-1098; (b) S. Shaik, R. Ramanan, D. Danovich and D. Mandal, Chem. Soc. Rev., 2018, 47, 5125-5145.

13 For selected effects of EEF upon reactions, see: (a) S. Shaik, S. P. de Visser and D. Kumar, J. Am. Chem. Soc., 2004, 126, 11746-11749; (b) H. Hirao, H. Chen, M. A. Carvajal, Y. Wang and S. Shaik, J. Am. Chem. Soc., 2008, 130, 33193327; (c) R. Ramanan, D. Danovich, D. Mandal and S. Shaik, J. Am. Chem. Soc., 2018, 140, 4354-4362; (d) W. Z. Lai, H. Chen, K. B. Cho and S. Shaik, J. Phys. Chem. Lett., 2010, 1, 2082-2087; (e) Z. F. Wang, D. Danovich, R. Ramanan and S. Shaik, J. Am. Chem. Soc., 2018, 140, 13350-13359; $(f)$ K. Bhattacharyya, S. Karmakar and A. Datta, Phys. Chem. Chem. Phys., 2017, 19, 22482-22486.

14 Y. Pocker and R. F. Buchholz, J. Am. Chem. Soc., 1970, 92, 4033-4038.

15 R. Meir, H. Chen, W. Z. Lai and S. Shaik, ChemPhysChem, 2010, 11, 301-310.

16 A. C. Aragonès, N. L. Haworth, N. Darwish, S. Ciampi, N. J. Bloomfield, G. G. Wallace, I. Diez-Perez and M. L. Coote, Nature, 2016, 531, 88-91.

17 (a) B. Xu and N. J. Tao, Science, 2003, 301, 1221-1223; (b) W. Haiss, R. J. Nichols, H. van. Zalinge, S. J. Higgins, D. Bethella and D. J. Schiffrin, Phys. Chem. Chem. Phys, 2004, 6, 4330-4337; (c) W. Haiss, C. S. Wang, I. Grace, A. S. Batsanov, D. J. Schiffrin, S. J. Higgins, M. R. Bryce, C. J. Lambert and R. J. Nichols, Nat. Mater., 2006, 5, 9951002.

18 For hydroboration reactions by action of EEF, see: (a) M. X. Zhang, F. Y. Zhang, H. L. Xu and Z. M. Su, New J. Chem., 2018, 42, 18402-18408; (b) M. X. Zhang and H. L. Xu, Phys. Chem. Chem. Phys., 2019, 21, 18-21.

19 For examples of Markovnikov hydroboration of alkenes, see: (a) P. K. Verma, A. S. Sethulekshmi and K. Geetharani, Org. Lett., 2018, 20, 7840-7845; (b) J. R. Smith, B. S. L. Collins, M. J. Hesse, M. A. Graham, E. L. Myers and V. K. Aggarwal, J. Am. Chem. Soc., 2017, 139, 9148-9151.

20 For introduction of B3LYP method, see: (a) A. D. Becke, $J$. Chem. Phys., 1992, 96, 2155-2160; (b) A. D. Becke, J. Chem. Phys., 1992, 97, 9173-9177; (c) A. D. Becke, J. Chem. Phys., 1993, 98, 5648-5652; (d) C. T. Lee, W. T. Yang and R. G. Parr, Phys. Rev. B: Condens. Matter Mater. Phys., 1988, 37, 785-789; (e) R. Krishnan, J. S. Binkley, R. Seeger and J. A. Pople, J. Chem. Phys., 1980, 72, 650-654.

21 A. V. Marenich, C. J. Cramer and D. G. Truhlar, J. Phys. Chem. B, 2009, 113, 6378-6396.

22 M. J. Frisch, G. W. Trucks, H. B. Schlegel, G. E. Scuseria, M. A. Robb, J. R. Cheeseman, G. Scalmani, V. Barone, B. Mennucci, G. A. Petersson, H. Nakatsuji, M. Caricato, X. Li, H. P. Hratchian, A. F. Izmaylov, J. Bloino, G. Zheng, J. L. Sonnenberg, M. Hada, M. Ehara, K. Toyota, 
R. Fukuda, J. Hasegawa, M. Ishida, T. Nakajima, Y. Honda, O. Kitao, H. Nakai, T. Vreven, J. A. Montgomery Jr, J. E. Peralta, F. Ogliaro, M. Bearpark, J. J. Heyd, E. Brothers, K. N. Kudin, V. N. Staroverov, T. Keith, R. Kobayashi, J. Normand, K. Raghavachari, A. Rendell, J. C. Burant, S. S. Iyengar, J. Tomasi, M. Cossi, N. Rega, J. M. Millam, M. Klene, J. E. Knox, J. B. Cross, V. Bakken, C. Adamo, J. Jaramillo, R. Gomperts, R. E. Stratmann, O. Yazyev, A. J. Austin, R. Cammi, C. Pomelli, J. W. Ochterski, R. L. Martin, K. Morokuma,
V. G. Zakrzewski, G. A. Voth, P. Salvador, J. J. Dannenberg, S. Dapprich, A. D. Daniels, O. Farkas, J. B. Foresman, J. V. Ortiz, J. Cioslowski and D. J. Fox, Gaussian 09, Revision D.01, Gaussian, Inc., Wallingford CT, 2013.

23 (a) T. Lu and F. W. Chen, J. Comput. Chem., 2012, 33, 580592; (b) T. Lu and F. W. Chen, J. Theor. Comput. Chem., 2012, 11, 163-183.

24 G. Merino, V. I. Bakhmutov and A. Vela, J. Phys. Chem. A, 2002, 106, 8491-8494. 